\title{
Low-cost, in-liquid measuring system using a novel compact oscillation circuit and quartz-crystal microbalances (QCMs) as a versatile biosensor platform
}

\author{
Stefan Beißner ${ }^{1, *}$, Jan-Wilhelm Thies ${ }^{2,4,{ }^{*}}$, Christopher Bechthold ${ }^{1, *}$, Philipp Kuhn ${ }^{3}$, \\ Bettina Thürmann $^{2}$, Stefan Dübel ${ }^{3}$, and Andreas Dietzel ${ }^{2,4}$ \\ ${ }^{1}$ Hochschule Hannover, Fakultät für Elektro- und Informationstechnik, Ricklinger Stadtweg 120, \\ 30459 Hannover, Germany \\ ${ }^{2}$ TU Braunschweig, Institute of Microtechnology, Alte Salzdahlumer Str. 203, 38124 Braunschweig, Germany \\ ${ }^{3} \mathrm{TU}$ Braunschweig, Institute of Biochemistry, Biotechnology and Bioinformatics, Spielmannstr. 7, \\ 38106 Braunschweig, Germany \\ ${ }^{4}$ TU Braunschweig, Center of Pharmaceutical Engineering (PVZ), Franz-Liszt-Straße 35A, \\ 38106 Braunschweig, Germany \\ * These authors contributed equally to this work.
}

Correspondence to: Stefan Beißner (stefan.beissner@hs-hannover.de) and Jan-Wilhelm Thies (j.thies@tu-braunschweig.de)

Received: 16 January 2017 - Revised: 25 July 2017 - Accepted: 31 August 2017 - Published: 9 October 2017

\begin{abstract}
Quartz-crystal microbalances (QCMs) are commercially available mass sensors which mainly consist of a quartz resonator that oscillates at a characteristic frequency, which shifts when mass changes due to surface binding of molecules. In addition to mass changes, the viscosity of gases or liquids in contact with the sensor also shifts the resonance but also influences the quality factor ( $Q$-factor). Typical biosensor applications demand operation in liquid environments leading to viscous damping strongly lowering $Q$-factors. For obtaining reliable measurements in liquid environments, excellent resonator control and signal processing are essential but standard resonator circuits like the Pierce and Colpitts oscillator fail to establish stable resonances. Here we present a lowcost, compact and robust oscillator circuit comprising of state-of-the-art commercially available surface-mount technology components which stimulates the QCMs oscillation, while it also establishes a control loop regulating the applied voltage. Thereby an increased energy dissipation by strong viscous damping in liquid solutions can be compensated and oscillations are stabilized. The presented circuit is suitable to be used in compact biosensor systems using custom-made miniaturized QCMs in microfluidic environments. As a proof of concept we used this circuit in combination with a customized microfabricated QCM in a microfluidic environment to measure the concentration of C-reactive protein (CRP) in buffer (PBS) down to concentrations as low as $5 \mu \mathrm{g} \mathrm{mL}^{-1}$.
\end{abstract}

\section{Introduction}

Quartz-crystal microbalances (QCMs) are mass sensors that are used for tasks like the thickness control during thinfilm deposition and nowadays a lot of biosensor applications are already reported in literature. Good surveys are offered by the reviews of Becker, Cooper and Speight (Becker and Cooper, 2011; Cooper and Singleton, 2007; Speight and Cooper, 2015). Such biosensor applications include detection and quantification of bacteria, protein-protein interactions, as well as protein adsorption, lipid-film formation, and cell adhesion to just name a few. The QCM technology is already well-known for some decades. Nevertheless, new approaches to shift the limit of detection into the sub-nanogram region were developed recently. With the utilization of nanoparticles, sensitivity enhancements can be achieved, making the QCM applicable to new measurements that were not possi- 
ble before. An overview of these improvements is given in Skládal (2016).

For applying QCM sensors in the gas phase, a lot of rather simple and reliable oscillator circuits are available. However, typical biosensor applications are carried out in a liquid environment where viscous damping leads to a significant loss in the $Q$-factor and therefore requires specific solutions. A good overview of available interface circuits for QCMs can be found in Lucklum and Eichelbaum (2007). To compensate the damping effect, Borngräber (2001) already developed an oscillating circuit with an integrated control loop in 2001 with the goal to keep the amplitude of the oscillation constant, even in more viscous environments like water.

After a short introduction into the problem of QCMs under high damping, we will introduce a new circuit built with surface mounted devices (SMDs) based on the Borngräber circuit utilizing commercially available SMDs leading to a very compact design resulting in smaller stray capacitances and inductances and thus to a very stable behaviour. To prove its functionality, measurements of artificial samples with Creactive protein were carried out with the new circuitry and microfabricated QCM devices.

\section{Origins of QCM frequency shifts}

QCM sensors belong to the group of thickness-shear mode resonators. Their measurement principle is based on the piezoelectric properties of quartz. Typically an electrode is attached to each of the two sides of a quartz-crystal disk. An alternating electrical potential is applied, leading to mechanical deflections and finally oscillation is obtained. The oscillation mode and its characteristic resonant frequency $f_{0}$ are defined by the crystallographic orientation of the blank, its thickness $t_{\mathrm{Q}}$, and properties of quartz such as it's density $\left(\rho_{\mathrm{q}}=2.65 \times 10^{3} \mathrm{~kg} \mathrm{~m}^{-3}\right)$ and elastic modulus $\left(\mu_{\mathrm{Q}}=\right.$ $2.947 \times 10^{11} \mathrm{~g} \mathrm{~cm}^{-1} \mathrm{~s}^{-2}$ ) (Rabe et al., 2000).

$f_{0}=\frac{1}{2 \cdot t_{\mathrm{Q}}} \sqrt{\frac{\mu_{\mathrm{Q}}}{\rho_{\mathrm{Q}}}}$

The oscillation frequency also depends on the mass of the oscillating object. This dependency can be utilized as a sensor principle where an additional mass $m_{\mathrm{S}}$, which becomes bound to the sensor surface, is transduced into a frequency drop $\Delta f_{\mathrm{m}}\left(A_{\mathrm{el}}\right.$, area of electrodes; $t_{\mathrm{Q}}$, thickness of the quartz plate) (Sauerbrey, 1959):

$\Delta f_{\mathrm{m}}=-f_{0} \frac{\Delta m_{\mathrm{S}}}{\rho_{\mathrm{Q}} \cdot t_{\mathrm{Q}} \cdot A_{\mathrm{el}}}$.

This correlation is only strictly valid within vacuum and for small mass increases $(<2 \%)$. For operation with liquid samples typically only one side of the QCM is in direct contact with the liquid solution, while the other side is in contact with air to prevent short circuits. The contact with liquid media leads to drastic changes which are not accounted

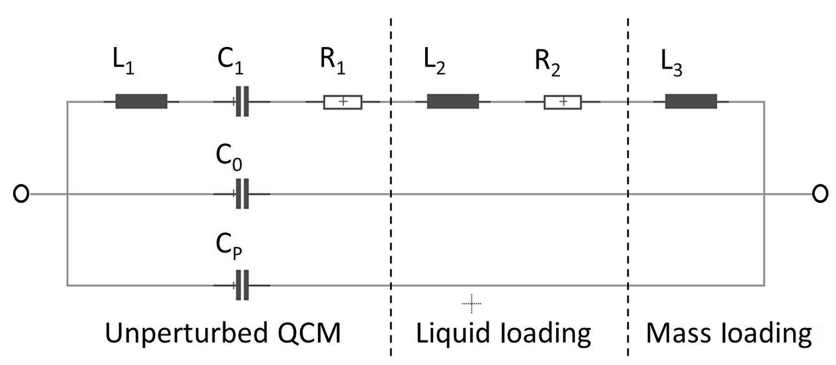

Figure 1. Modified Butterworth-van Dyke circuit after Martin et al. (1991).

for in Eq. (2). In liquid, the damping of the oscillation increases rapidly. Kanazawa and Gordon found a relation between the change of oscillating frequency and the properties of the surrounding liquid media. They developed a physical model encompassing the coupling of the standing shear wave in the lossless quartz crystal with the damped propagating shear wave within the fluid as follows $\left(\eta_{\mathrm{L}}\right.$ : liquid viscosity, $\mathrm{kg} \mathrm{s}^{-1} \mathrm{~m}^{-1} ; \rho_{\mathrm{L}}$ : liquid density, $\mathrm{kg} \mathrm{m}^{-3}$ ) (Kanazawa and Gordon, 1985):

$\Delta f=-f_{0}^{\left(\frac{3}{2}\right)} \cdot \sqrt{\frac{\eta_{\mathrm{L}} \cdot \rho_{\mathrm{L}}}{\pi \cdot \mu_{\mathrm{Q}} \cdot \rho_{\mathrm{Q}}}}$.

The resonant frequency depends on the product of the fluids viscosity $\left(\eta_{\mathrm{L}}\right)$ and density $\left(\rho_{\mathrm{L}}\right)$ thus heavy, viscous environments cause a frequency drop $\Delta f$ against measurement in a vacuum environment. Since the resonant frequency is on one hand affected by mass loading and on the other hand by liquid loading, measurements of the resonant frequency alone cannot distinguish between changes in surface mass from changes in solution properties.

Martin et al. (1991) expanded the Butterworth-Van Dyke equivalent circuit for quartz-oscillators with elements that are related to physical properties of the additional mass layer and the contacting liquid (Fig. 1).

The capacities $C_{0}$ and $C_{1}$, the inductivity $L_{1}$, and the resistor $R_{1}$ describe properties of the quartz, while $C_{\mathrm{p}}$ is an additional term for a parasitic capacitance of the test fixture. The dielectric quartz material and parasitic contributions of the wiring and the crystal holder are combined into the static capacitance $C_{0}$. The initial mass is taken into account by $L_{1}$, while the mechanical elasticity of the quartz is reflected by $C_{1}$. Finally energy losses due to internal friction, mechanical damping of the quartz, and the crystal holder are merged into $R_{1}$.

While a mass increase can be expressed as additional inductance term $L_{3}$, a liquid loading is taken into account by adding an additional resistance term $R_{2}$ for energy dissipation and an inductive term $L_{2}$ for the additional mass layer of the fluid that is in direct contact with the resonator (Baltes et al., 2001). 
According to Lucklum et al. (2007) the series resonant frequency $f_{\mathrm{r}}$ can, in the absence of liquid and mass loading, be calculated for small $R_{1}$ as the following:

$f_{\mathrm{r}} \approx \frac{1}{2 \pi} \cdot \sqrt{\frac{1}{L_{1} \cdot C_{1}}+\frac{R_{1}^{2} \cdot C_{0}}{L_{1}^{2} \cdot C_{1}}}$

For biosensor applications, the sensor has to be functionalized with a sensitive coating and be in contact with an analyte containing buffer solution. In this case, the resonant frequency has to take $R_{2}, L_{2}$, and $L_{3}$ into account too. $L_{1}$ and $R_{1}$ are then exchanged in equation 4 with the following terms:

$R_{1} \rightarrow R_{1}+R_{2}$,

$L_{1} \rightarrow L_{1}+L_{2}+L_{3}$.

For a QCM, the $Q$-factor, which describes the quality of a resonator, can be defined as the following (Rabe, 2003):

$Q=\frac{1}{R_{1}} \cdot \sqrt{\frac{L_{1}}{C_{1}}}$.

Since higher liquid loading leads to an increased resistance $R_{1}$ the quality factor decreases and the resonator has to be supplied with more energy to maintain the oscillation. Oscillating circuits that are not able to compensate this $Q$ factor decline and are not suitable for QCM measurements in liquids since they will become very unstable or even stop oscillating.

A detailed discussion of viscoelastic effects in QCM measurements can be found in Johannsmann (2007).

\section{Microfluidic chip with integrated QCM devices}

QCM devices were fabricated from $120 \mu \mathrm{m}$ thick Quartz blanks (from KRYSTALY, Hradec Králové, a.s, Czech Republic). Thin membranes (thickness around $78 \mu \mathrm{m}$ ) were obtained by a lithographic process including wet etching. Only one side of the blank was machined, while the other side was covered with a sacrificial gold layer. By this method, the polished surface of the blank could be protected. After separation of the membrane elements by dicing, single, round membranes with a thick mechanically stable outer ring are obtained. These finished quartz membranes are then embedded into a microfluidic chip (Fig. 2) made from polydimethylsiloxane (PDMS) (Sylgard 184, Dow Corning). Electrical contact was achieved by applying conductive varnish (Eccobond 59C, Emerson \& Cumning). A printed circuit board (PCB) is used as a carrier and allows for connection to the oscillation circuit. The detailed fabrication process can be found in Thies et al. (2017).

A big advantage of this chip compared to conventional round QCM membrane designs is the attachment of the microfluidic housing to a thick brim around the membrane. Due

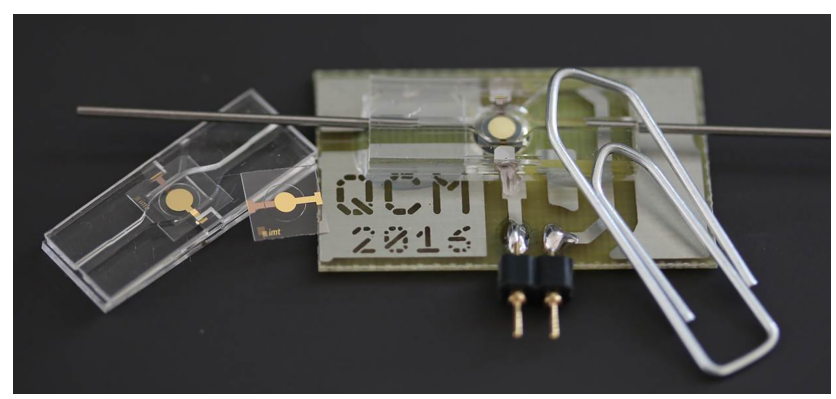

Figure 2. Microdevices used in our studies at different integration levels: microfluidic chip containing a QCM (left), small QCM obtained after dicing the microstructured quartz substrates (middleleft), the ready-to-use microfluidic chip mounted on a PCB with a paper clip for comparison of sizes (right).

to this brim, damping of the oscillator by the attached housing is reduced. Furthermore, the PDMS flow cell only has a small dead volume, which reduces liquid dispersion effects, making it more suitable for biosensing. To avoid crosscontamination in point-of-care applications, the whole system is designed as disposable.

\section{Quartz oscillator circuits}

As discussed above, the overall quartz impedance can be measured but not its individual components. The impact of different environments on the electrical behaviour of integrated QCMs has been measured and is shown in Fig. 3.

Here the effect of different liquid media and filling levels over the QCM becomes visible: with increasing filling level and increasing viscosity the magnitude of the impedance decreases, while the curve for the phase angle broadens around resonance. For these measurements, we used in-house fabricated QCM devices with a PDMS housing and applied different media. At first the device was operated in air. Afterwards we began to fill it with deionized water until it was filled completely. We also used Miglyol 812 (Caesar \& Loretz GmbH, viscosity $30 \mathrm{mPas})$. The measurements were performed using a network analyser (Hewlett Packard E5100A) and are obtained for the same QCM which was rinsed with deionized water between the measurements. This can illustrate that evaluation circuits, which rely on a high $Q$-factor and sharply defined phase angles at resonance may not be suited for measurements with QCM in liquid media.

Instead of using an expensive network analyser, a compact and low-cost circuitry for the evaluation of a QCM in a microfluidic biosensor environment is highly preferred for compact and easy-to-use biosensing applications. Even though LC- and RC-oscillators are easily available, many circuits for resonance frequency determination use an internal vibrating quartz because the mechanically resonating quartz usually shows a superior $Q$-factor and temperature stability 

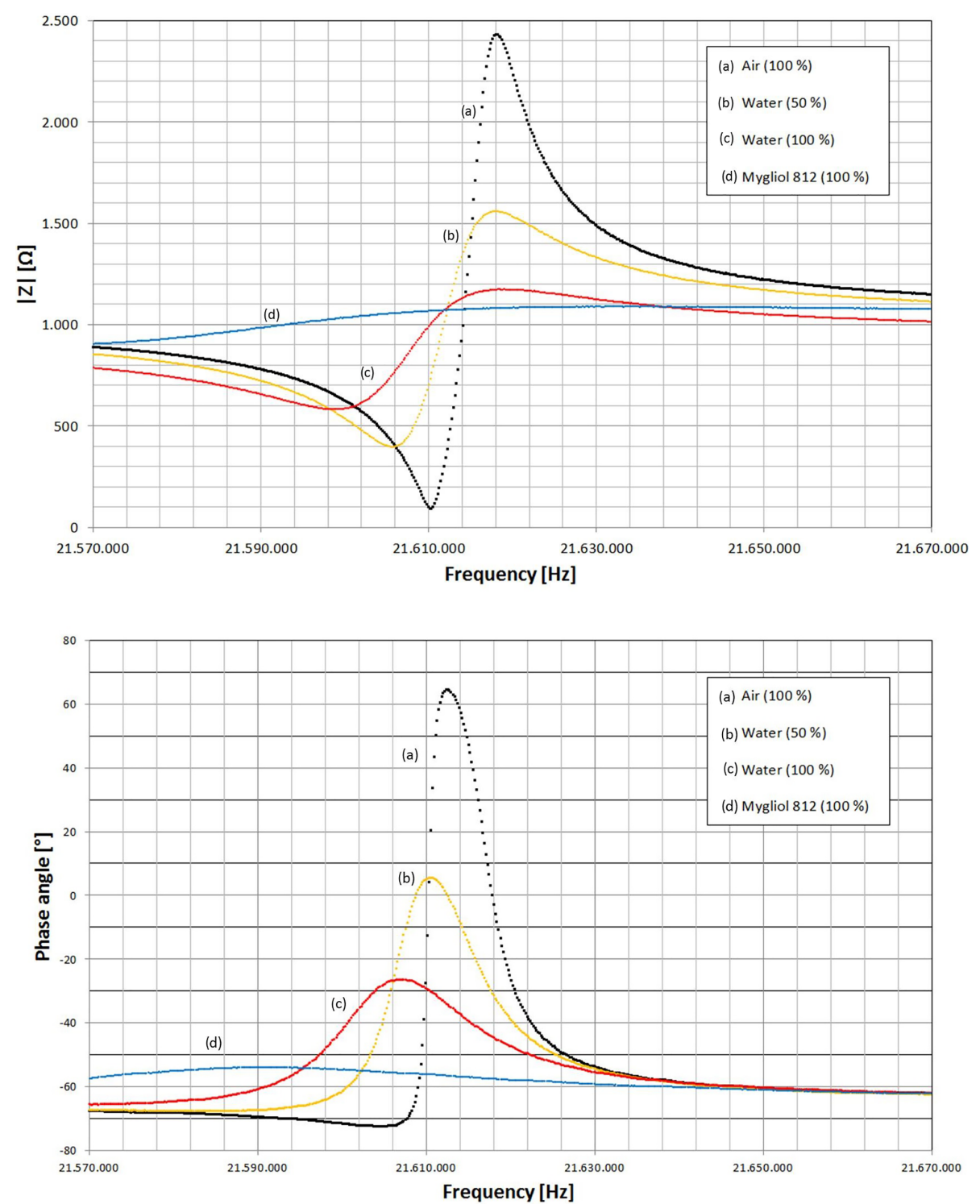

Figure 3. Changes in amplitude (top panel) and phase angle (bottom panel) for different media as obtained from microfluidic chips with integrated QCM using a network analyser. The percentage information indicates the filling level of the microfluidic chip. Where $100 \%$ indicates a completely filled chip, while $50 \%$ describes a half-filled chip.

compared to purely electronically resonating LC- and RCcircuits.

A Pierce oscillator (Fig. 4) is the standard circuit to generate the clock pulse in digital circuits. The quartz crystal $(X)$ forms a $\pi$-filter with the capacitors $C_{1}$ and $C_{2}$. The filter is applied in the feedback loop of the inverter $U_{1}$, which makes the circuit oscillate on the series resonance of the quartz. The second inverter $\left(U_{2}\right)$ amplifies the signal and turns it into a square waveform.

An oscillating circuit for a QCM has to fulfil some additional specifications. If the quartz should be able to oscillate in gas as well as in liquid, the circuit has to be able to deal with different damping factors. Nevertheless the Pierce os- cillator has been recently reported for use as a cheap, compact and simple to implement QCM-measurement platform (openqcm, 2016) but easily becomes unstable in viscous liquids with strong damping. The measurements we present in Sect. 5 have also been carried out with the Pierce oscillator circuit from the openQCM project (openqcm, 2016). While our very small QCMs were oscillating with this circuit in air, the Pierce oscillator failed to maintain the oscillation in liquids, preventing the measurement of binding events completely.

In 1997, Rösler (1997) reported an oscillator circuit for quartz sensors. The functional principle of the circuit can be explained by Fig. 5. The circuit in Fig. 5 consists of two com- 


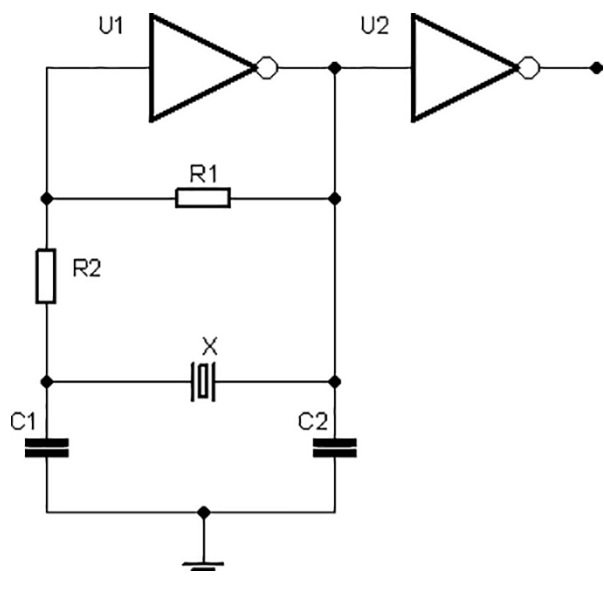

Figure 4. Circuit diagram of a Pierce oscillator circuit.

mon emitter circuits which are coupled by a capacitor. The quartz sensor (xtal) is placed at the emitter of the first transistor $Q 1$. Since the quartz maximizes the amplification of the first common emitter circuit at its series resonance frequency, it makes the entire circuit oscillate at this frequency. To overcome the shortcomings of discrete transistors like nonlinearities, parasitic capacitances, and unstable operating points, Rösler realized his circuit using an operational transconductance amplifier (OTA). The OTA contains a so-called "diamond transistor". The diamond transistor is a circuit which can be seen as a nearly ideal transistor with internally defined operating points and very small temperature drift. Basically, the diamond-transistor is used to replace the transistor $Q 1$ in Fig. 5. Even this circuit is not very stable at high damping and does not measure the damping of the QCM but only measures the frequency of the oscillation.

Since frequency is influenced by the mass change on the surface of the quartz and by the viscosity of the surrounding liquid, a change in viscosity cannot be distinguished from a mass change (Borngräber, 2001). Based on Röslers' (1997) OTA circuit, Borngräber (2001) proposed an oscillating circuit which contains a control loop that keeps the amplitude of the oscillation stable. As a result, the actuating variable of this control loop can be used to determine the damping effect of the viscosity of the surrounding liquid.

For this purpose, a complex calibration process is necessary. This process involves the calibration of the oscillation circuit with different damped QCMs and the determination of the undamped measurement QCM with a network analyser. Afterwards, the results are calculated and a corrected series resonant frequency and series impedance are obtained. A detailed overview of this complex calibration method can be found in Borngräber (2001). Since 1997, a lot of development has been done and nowadays the QCM with dissipation monitoring (QCM-D) technique is available (Dixon, 2008). This technique allows to measure not only the bound mass but also the energy dissipation, giving a mea-

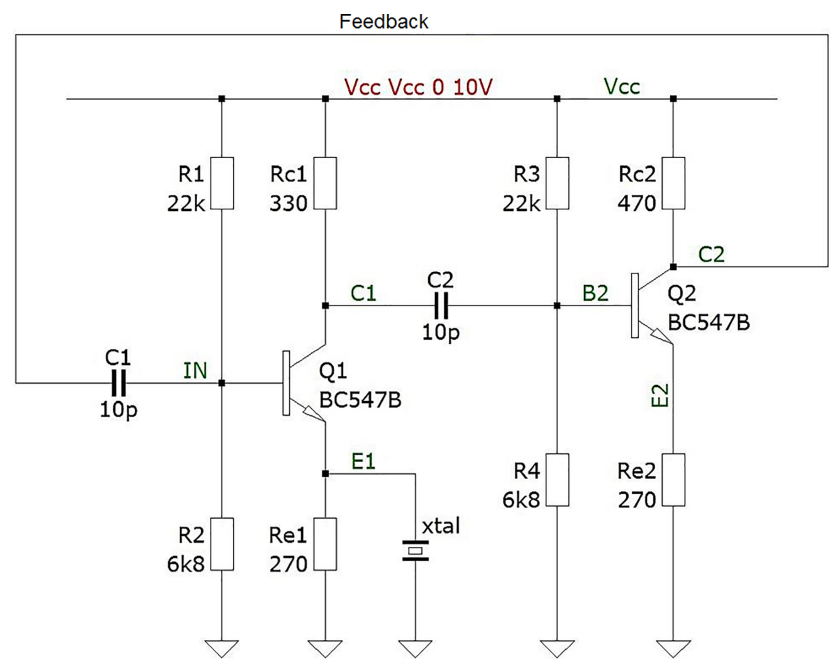

Figure 5. Functional principle of the oscillator circuit by Rösler (1997). This figure is the basis to explain the oscillator circuit; the actual circuit is more sophisticated using an operational transconductance amplifier (OTA) instead of discrete transistors.

sure for the rigidity of the bound film. Here we focus on stabilizing the oscillation. The obtained frequency shift might not be enough for studying film properties but is sufficient for biomolecule detection and quantification in point-of-care measurements. The presented circuit is considerably cheaper than commercial QCM-D equipment.

\section{The new robust, compact and low-cost QCM circuit}

Based on the Borngräber circuit, this new circuit is using a control loop with a PI-controller to keep the amplitude of the oscillation of the QCM sensor stable in spite of different damping factors and thereby compensating for the $Q$-factor decline. The block diagram of this circuit is shown in Fig. 6 .

The new circuit is using the operational transconductance amplifier OPA860 in SMD technology. While an ordinary operational amplifier amplifies an input voltage to an output voltage, an OTA like the OPA860 amplifies an input voltage to an output current. It can be seen as a voltage controlled current source. Furthermore, the OPA860 contains a unity gain buffer, which is used in our circuit. The circuit is shown in Figs. 7 and 8.

The reference value for the amplitude of the oscillation is set by $P_{1}$. The measured value is the DC-equivalent of the controlled variable. The PI-controller $\left(\mathrm{IC}_{2}\right)$ keeps the measured value equal to the reference value. Thus, the amplitude is kept on the same level, even if the damping of the oscillator is significantly different.

The circuit is assembled using SMD components on a $53 \mathrm{~mm} \times 66 \mathrm{~mm}$ PCB (Fig. 9). Due to smaller stray capacitances and smaller inductances of the tracks on the board, the SMD board is significantly more stable than older and less- 


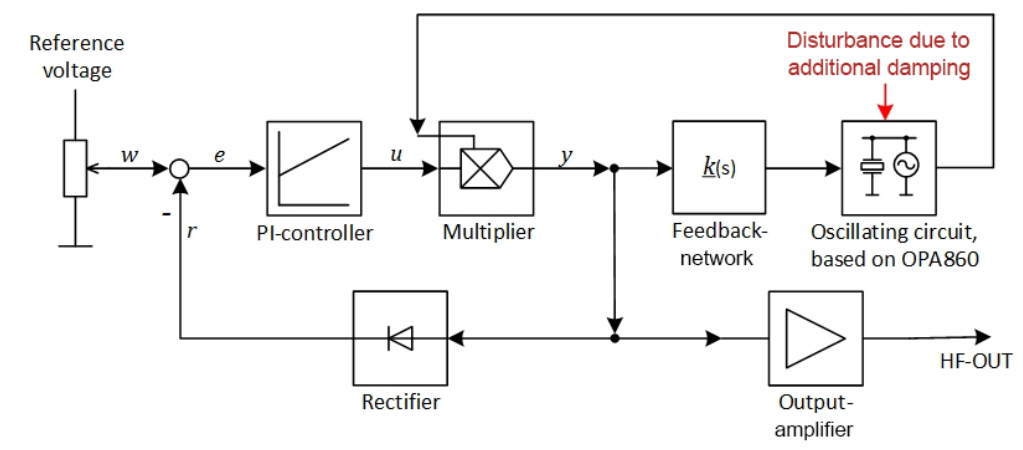

Figure 6. Block diagram of the new compact and low-cost circuit.

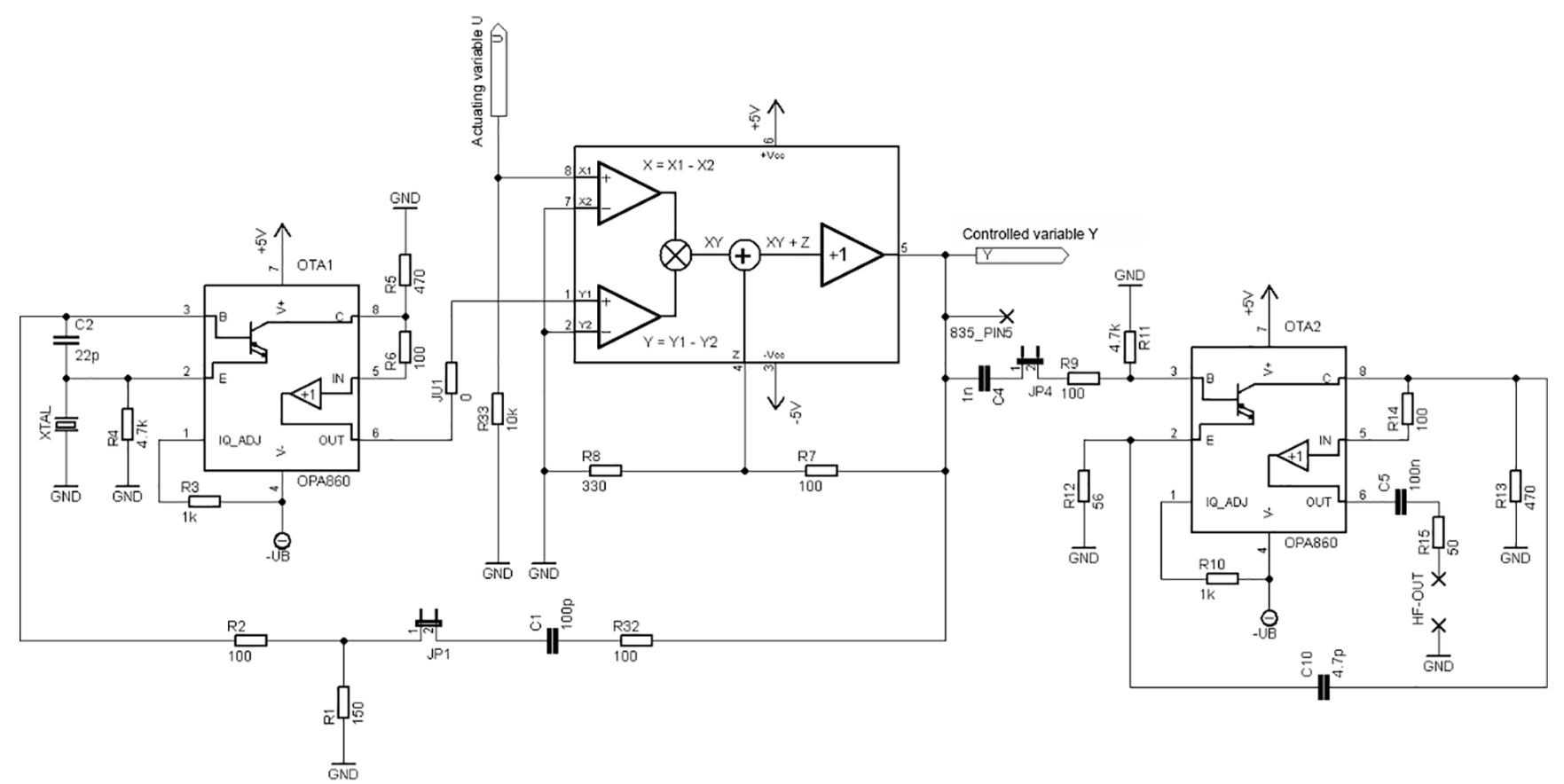

Figure 7. Front-end of our QCM circuit with the oscillator (built with the quartz XTAL and OTA1), the analogue multiplier AD835, the feedback-network (R1, R2, C1, and R32) and the output amplifier (around OTA2), which generates the HF-OUT-signal. The controlled variable $Y$ is the amplitude of the oscillation.

compact Borngräber circuits using through-hole technology components.

For further research, the PCB layout is available online in the Supplement.

\section{Measurements and results}

A measured frequency drop can provide information about chemical and biological processes taking place at the gold electrode of the QCM. To receive a specific sensor response, the electrode surface has to be modified to selectively react with the target analyte. In a previous work, Balck used the Borngräber circuit to measure the concentration of human C-reactive protein (CRP) (Balck et al., 2011). CRP is currently the most important molecular marker for acute in- flammation in the human blood serum. As with other acutephase proteins, its concentration increases in response to an inflammation. While healthy human beings have a CRP level in serum between 5 and $10 \mathrm{mg} \mathrm{L}^{-1}$, it can increase above $200 \mathrm{mg} \mathrm{L}^{-1}$ in severe inflammation events, like in bacterial sepsis, pneumonitis, pancreatitis or active rheumatoid arthritis. It discriminates between viral and bacterial infections and therefore is very helpful for the treatment decision, e.g. whether antibiotics should be given in cases of fever with unclear etiology. Small and affordable CRP biosensors therefore, would offer a much quicker diagnosis at the "point of care" and thus allow better treatment. Here, the CRP concentration of different samples is measured for demonstration of the functionality of the new circuit in conjunction with our microfluidic chip with our embedded custom-made QCM, as 


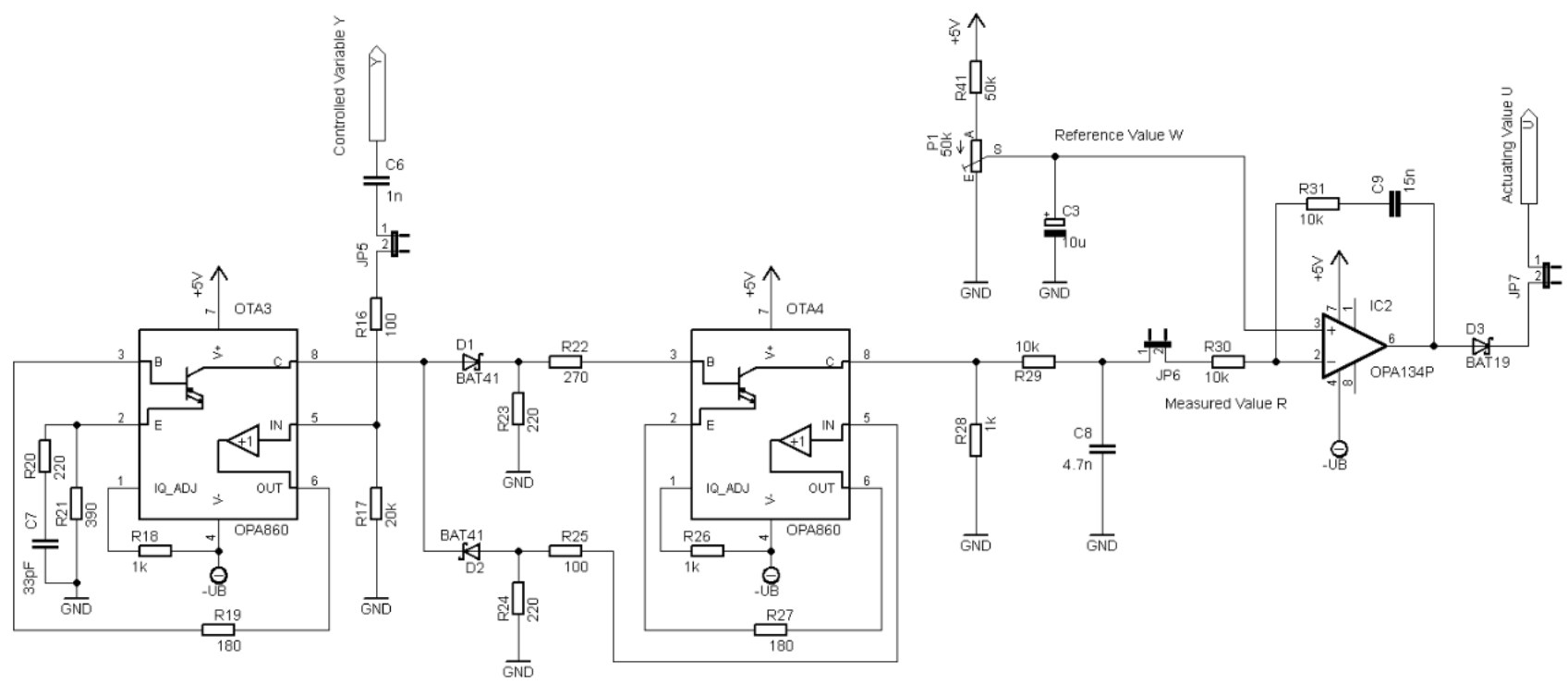

Figure 8. Back-end of our QCM circuit with the reference value $W$, the PI-controller IC2, and the rectifier (around D1, D2, OTA3, and OTA4) that generates the measured value $R$.
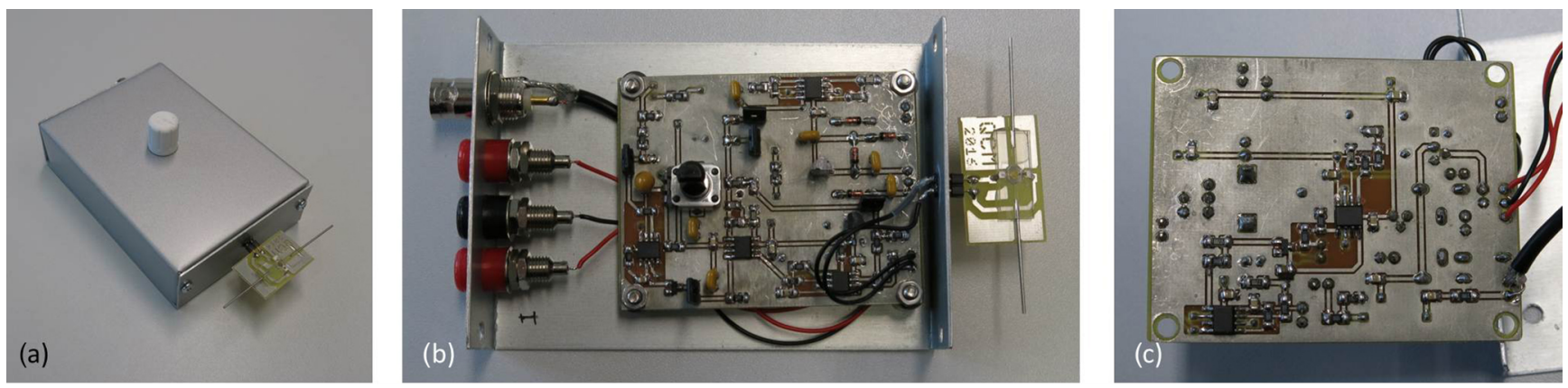

Figure 9. The SMD board $(53 \mathrm{~mm} \times 66 \mathrm{~mm})$ of the circuits shown in Figs. 7 and 8: (a) shielded housing with attached QCM device, (b) upper, and (c) backside of the oscillator circuit board. The knob is connected to $P_{1}$ and is used to set the reference value of the amplitude of the oscillation.

shown in Fig. 2. For preparation of the chip we first apply a self-assembled monolayer (SAM), which directly attaches to the gold electrode. This SAM is made in two steps. First the chip is filled with a cysteamine solution (Sigma-Aldrich, $20 \mathrm{mmol} \mathrm{L}^{-1}$ in deionized water) for $12 \mathrm{~h}$. During this time, the thiol groups of the cysteamine bind to the gold electrode. Afterwards the chip is filled with glutaraldehyde (SigmaAldrich, 2.5 vol. \% in PBS) and incubated for an additional 2 hours. Together, cysteamine and glutaraldehyde react and form a very reactive and dense, self-assembling monolayer (Wirde et al., 1999).

During the measurements the evaluation circuit has been connected to a frequency counter (53220A, Agilent Technologies) and a laboratory power supply (E3631A, Agilent Technologies). The frequency counter readout was evaluated with LabView (LabView 2014, National Instruments). The buffer solution (phosphate buffered saline solution, PBS;
Sigma-Aldrich, $\mathrm{pH} 7.4,0.01 \mathrm{M})$ was continually pumped with a neMESYS syringe pump (Cetoni $\mathrm{GmbH}$ ). Samples were applied with an injection valve (model 7125, Rheodyne LLC) into the buffer solution. The measurement was started after a stable baseline was formed (see Fig. 10). For the detection of CRP (Bio-Rad, Native Human C-Reactive Protein 1707-2029), recombinant antibodies (scFv-Fc) generated in vitro by antibody phage-display, with binding properties specially engineered to fit to the measurement principle (Al-Halabi et al., 2013) have to be bound to the SAM. This step defines which substances will be measured with the QCM, as such recombinant antibodies made by in vitro evolution can be obtained to specifically detect almost any protein and even many non-proteinous biomolecules (Frenzel et al., 2012; Dübel et al., 2010). The attachment of the antibody leads to a first drop of the resonant frequency of around $280 \mathrm{~Hz}$. 


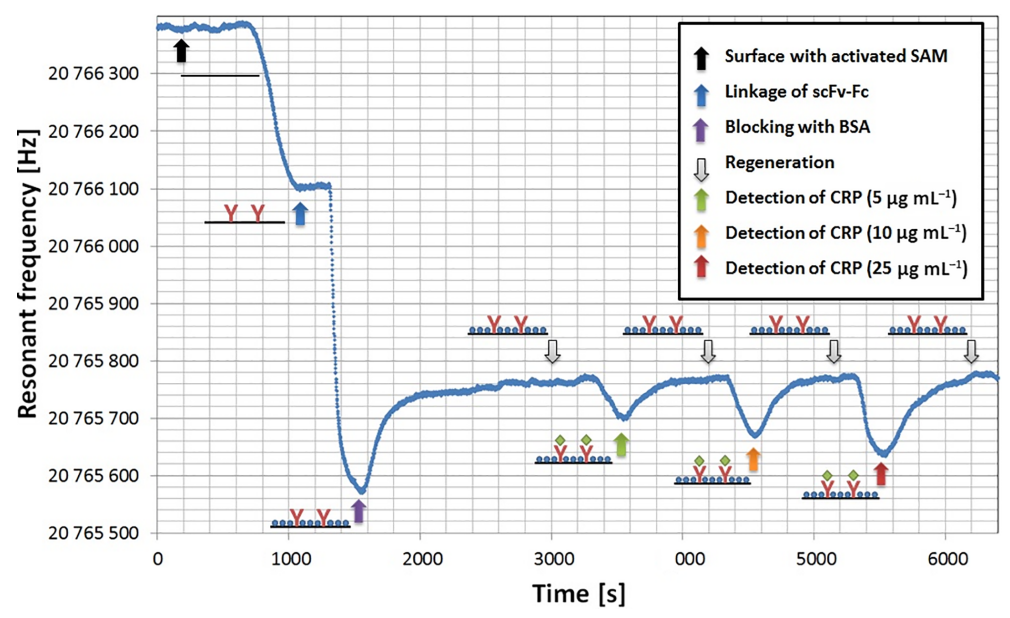

Figure 10. Continuous resonant frequency measurement: after preparation of the chip with antibody and blocking reagent (BSA), different CRP concentrations were applied.

Free binding sites that are not covered by the anti-CRP antibodies have to be blocked to prevent baseline drift by later unspecific binding of other substances to the QCM. For this purpose, bovine serum albumin (BSA; Sigma-Aldrich, $1 \% \mathrm{w} / \mathrm{v}$ in deionized water) was used. The blocking event resulted in a large frequency drop $(\sim 525 \mathrm{~Hz})$, which was reversible to some extent $(\sim 210 \mathrm{~Hz})$. The irreversible drop of resonant frequency is the signal indicating the BSA attachment to unused covalent reaction sites of the SAM. The reversible part is supposed to result from washing away the excess of non-covalently attached BSA and the difference in viscosity of PBS and the BSA solution. After blocking, the chip was ready for CRP measurements. The repeated application of samples containing CRP in different concentrations resulted in concentration-dependent frequency drops. Here we applied 5, 10, and $25 \mu \mathrm{g} \mathrm{mL} \mathrm{m}^{-1} \mathrm{CRP}$. Due to the purposely designed kinetic properties of the recombinant anti-CRP antibody, the dissociation reaction, or in other words the release of the CRP from the sensor, took place within minutes. This behaviour allows for repetitive measurements without the need for special regeneration steps. It is sufficient to just purge the chip with buffer solution to prepare it for another measurement. This allows for calibration and hence quantification.

Since the same PBS was used in all samples, which contained only small quantities of different substances, the damping from samples can be considered as constant. This assumption is not valid if the damping through an unknown sample leads to an additional contribution to the frequency change, which can be compensated for by calibration. This is what appears in Fig. 10 when the BSA blocking solution is applied. The original frequency drop is $\sim 525 \mathrm{~Hz}$ but to some extent reversible $(\sim 210 \mathrm{~Hz})$. After this step, it is known that a $1 \% \mathrm{w} / \mathrm{v}$ BSA solution results in a reversible frequency drop of around $210 \mathrm{~Hz}$ for the QCM used here. If this would be used as calibration step and another BSA sample would be applied, then the reversible frequency drop through damping could be determined. The irreversible part of the BSA sample is the part of surface blocking which would not occur again. Since the CRP samples do not show such a behaviour, the damping is considered constant here.

However, saturation occurs when most antibody binding sites are already populated with antigen and the relationship between antigen concentration and sensor response is no longer linear. This effect is also found for other antibody-based measuring methods and further explained in Wild (2013).

In previous works (Balck et al., 2011), CRP concentrations down to $250 \mu \mathrm{g} \mathrm{mL}^{-1}$ could be detected. In comparison, our experiments (Fig. 10) show a sensitivity 50 times higher, which in part can result from the new very stable control circuit but also can be facilitated by a different biochemical assay that was used here.

There are other competing techniques to measure CRP concentrations. One established and widely used technique is the enzyme-linked immunosorbent assay (ELISA). This method uses antibodies and a colour or fluorescence change to identify and quantify a substance. For this, usually one antibody is bound to a microlitre plate. The sample is injected and after a certain incubation time, it is washed. A second antibody, binding to a different epitope of the analyte, is added and after incubation the sample chamber is washed again. Depending on the assay, a fluorescence label or a colour changing substance is added. Then the fluorescence response or the intensity is measured. If there is no analyte present, the second antibody and the fluorescence label or colour chemistry are washed away and as a result, no signal response is detected. If the antigen is present, a measurement response correlating with the concentration of the antigen is received and the amount of target analyte quantified. Commercially 
available CRP ELISA kits (Thermo Fisher Scientific Inc., 2017; Enzo Life Sciences Inc., 2017; Creative-Diagnostics, 2017) are able to quantify CRP samples with a detection limit down to some nanograms or even picograms of CRP per millilitre. Despite their superior limit of detection, ELISA protocols are not suitable as biosensors because their protocols take hours before a result is provided. They also lack the possibility to extract time dependent information like binding kinetics. Additionally, the saturation effect shown above can also appear in ELISA tests. This is why these tests nearly always have a standard curve in their data sheets or give an upper and lower limit of detection.

To increase the signal response of our measurement system, the antibody sandwich approach of ELISA can be utilized. By attaching a second antibody to the target analyte on the QCM, the applied mass is increased while the analyte concentration remains constant. This leads to a higher resonant frequency drop and allows us to measure concentrations that were not measurable before. We already presented this approach in Thies et al. (2017) and could increase our limit of detection to one microgram of CRP per millilitre of sample.

In terms of costs, the ELISA test and the QCM test might be comparable. The same antibodies or antibody sandwiches can be used. Commercially available QCM systems allow for reusing of the quartz crystal disc, while our flow cell is designed to be disposable. The components of the here presented circuit were assembled on an in-house fabricated PCB at low cost. Aside from the QCMs and the oscillation circuit, a pulsation-free pump and an injection valve are needed. This makes our system a very cheap measurement setup.

\section{Conclusions}

QCMs are widely known as sensitive (bio)sensors and recent attempts to enhance their sensitivity have been reported. While a lot of oscillation circuits exist, most are only suited for measuring in gaseous environments. Since typical pointof-care and biosensor applications take place in microfluidic environments, there is a need for cheap and compact oscillator circuits that are able to work under heavy damping. Here we present a robust and low-cost oscillator circuit with an integrated control loop for regulating the applied voltage. Thereby increased energy dissipation by strong viscous damping in liquid solutions can be compensated for and oscillations are stabilized. The circuit is assembled with lowcost components on a PCB. The small-sized SMD components together with the microfluidic chip lead to a compact design of the complete measuring system. Furthermore, the small size of the circuit leads to small stray capacitances and inductances making the device stable and robust. Using this new measuring system, we reliably detected concentrations of CRP down to $5 \mu \mathrm{g} \mathrm{mL}^{-1}$ relevant for point-of-care diagnostics. Due to the circuit's small size and low cost, it could be perfectly suited for implementation in point-of-care measurement systems and handheld applications.

Data availability. The measurement data for the QCM measurements and characterization are available in the Supplement.

\section{The Supplement related to this article is available online at https://doi.org/10.5194/jsss-6-341-2017-supplement.}

Competing interests. The authors declare that they have no conflict of interest.

Acknowledgements. Some authors gratefully acknowledge the financial support by the "Ministerium für Wissenschaft und Kultur" of Lower Saxony, Germany. The research is conducted within the program "Novel synthesis and formulation methods for poorly soluble drugs and sensitive biopharmaceuticals (SyFoBia)".

Edited by: Anita Lloyd Spetz

Reviewed by: two anonymous referees

\section{References}

Al-Halabi, L., Balck, A., Michalzik, M., Fröde, D., Büttgenbach, S., Hust, M., Schirrmann, T., and Dübel, S.: Recombinant antibody fragments allow repeated measurements of C-reactive protein with a quartz crystal microbalance immunosensor, mAbs, 5, 140-149, https://doi.org/10.4161/mabs.22374, 2013.

Balck, A., Michalzik, M., Al-Halabi, L., Dübel, S., and Büttgenbach, S.: Design and Fabrication of a Lab-on-a-chip for Pointof-care Diagnostics, Sensors Transducers, 127, 102-111, 2011.

Baltes, H., Hesse, J., and Korvink, J. G.: Sensors Update Vol. 9: A Comprehensive Survey, 1st Edn., WILEY-VCH, Weinheim, 2001.

Becker, B. and Cooper, M. A.: A Survey of the 2006-2009 Quartz Crystal Microbalance Biosensor Literature, J. Mol. Recognit., 24, 754-787, https://doi.org/10.1002/jmr.1117, 2011.

Borngräber, R.: Quarzresonatoren für flüssige Medien - Systemdesign und Anwendung, PhD Thesis, Institut für Mikro- und Sensorsysteme, Otto-von-Guericke-Universität Magdeburg, Magdeburg, 2001.

Cooper, M. A. and Singleton, V. T.: A Survey of the 2001 to 2005 Quartz Crystal Microbalance Biosensor Literature: Applications of acoustic physics to the analysis of biomolecular Interactions, J. Mol. Recognit., 20, 154-184, https://doi.org/10.1002/jmr.826, 2007.

Creative-Diagnostics: Human C-reactive Protein, Pentraxin-Related, CRP ELISA KitProd. No. DEIA217, http://img.creative-diagnostics.com/pdf/ DEIA217HumanCRP20ELISA20Kit.pdf, last access: 24 February 2017. 
Dixon, M. C.: Quartz Crystal Microbalance with Dissipation Monitoring: Enabling Real-Time Characterization of Biological Materials and Their Interactions, J. Biomol. Tech., 19, 151-158, 2008.

Dübel, S., Stoevesandt, O., Taussig, M. J., and Hust, M.: Generating recombinant antibodies to the complete human proteome, Trends Biotechnol., 28, 333-339, https://doi.org/10.1016/j.tibtech.2010.05.001, 2010.

Enzo Life Sciences Inc.: CRP (Human) ELISA kit ENZKIT102-0001, http://www.enzolifesciences.com/ENZ-KIT102/ crp-human-elisa-kit/, last access: 24 February 2017.

Frenzel, A., Fröde, D., Meyer, T., Schirrmann, T., and Hust, M.: Generating Recombinant Antibodies for Research, Diagnostics and Therapy Using Phage Display, Curr. Biotechnol., 1, 33-41, https://doi.org/10.2174/2211550111201010033, 2012.

Johannsmann, D.: Studies of Viscoelasticity with the QCM, in: Ser. Chem. Sens. Biosens., 5, Springer, Berlin, Heidelberg, 49-109, https://doi.org/10.1007/5346_024, 2007.

Kanazawa, K. K. and Gordon, J. G.: The oscillation frequency of a quartz resonator in contact with liquid, Anal. Chim. Ac., 175, 99-105, https://doi.org/10.1016/S0003-2670(00)82721-X, 1985.

Lucklum, R. and Eichelbaum, F.: Interface circuits for QCM sensors, in: Ser. Chem. Sens. Biosens., Piezoelectric Sensors, 1st Edn., edited by: Wolfbeis, O. S., Springer, Berlin, Heidelberg, 3-47, 2007.

Martin, S. J., Granstaff, V. E., and Frye, G. C.: Characterization of a quartz crystal microbalance with simultaneous mass and liquid loading, Anal. Chem., 63, 2272-2281, https://doi.org/10.1021/ac00020a015, 1991.

openqcm: http://openqcm.com/electronics, last access: 26 July 2016.

Rabe, J.: Miniaturisierte Quarzresonatoren und Arrays für AnalytikAnwendungen in Flüssigkeiten, $\mathrm{PhD}$ Thesis, Institut für Mikrotechnik, TU Braunschweig, Braunschweig, 2003.
Rabe, J., Büttgenbach, S., Zimmermann, B., and Hauptmann, P.: Design, Manufacturing, and Characterization of High-Frequency Thickness-Shear Mode Resonators, Proc. IEEE/EIA International Frequency Control Symposium, 9 June 2000, Kansas City, MO, USA, 2000.

Rösler, S. H.: Quarzresonator-Sensoren zum Nachweis chemischer Substanzen in Wasser, PhD Thesis, Otto von Guericke Universität Magdeburg, Magdeburg, 1997.

Sauerbrey, G.: Verwendung von Schwingquarzen zur Wägung dünner Schichten und zur Mikrowägung, Z. Physik, 155, 206-222, https://doi.org/10.1007/BF01337937, 1959.

Skládal, P.: Piezoelectric biosensors, TrAC Trends Anal. Chem., 79, 127-133, https://doi.org/10.1016/j.trac.2015.12.009, 2016.

Speight, R. E. and Cooper, M. A.: A survey of the 2010 Quartz Crystal Microbalance Literature, J. Mol. Recognit., 25, 451-473, https://doi.org/10.1002/jmr.2209, 2015.

Thermo Fischer Scientific Inc.: CRP Human ELISA Kit KHA0031, https://www.thermofisher.com/order/catalog/product/KHA0031, last access: 24 February 2017.

Thies, J.-W., Kuhn, P., Thürmann, B., Dübel, S., and Dietzel, A.: Microfluidic quartz-crystal-microbalance (QCM) sensors with specialized immunoassays for extended measurement range and improved reusability, Microelect. Eng., 179, 25-30, https://doi.org/10.1016/j.mee.2017.04.023, 2017.

Wild, D. G.: The Immunoassay Handbook, 4th Edn., Elsevier Science Ltd, Amsterdam, Boston, Heidelberg, London, New York, Oxford, Paris, San Diego, San Francisco, Singapore, Sydney, Tokyo, 2013.

Wirde, M., Gelius, U., and Nyholm, L.: Self-Assembled Monolayers of Cystamine and Cysteamine on Gold Studied by XPS and Voltammetry, Langmuir, 15, 6370-6378, https://doi.org/10.1021/la9903245, 1999. 